Research article

\title{
Functional identification of HugZ, a heme oxygenase from Helicobacter pylori
}

Ying $\mathrm{Guo}^{\dagger 1}$, Gang Guo ${ }^{\dagger 1}$, Xuhu Mao1, Weijun Zhang1, Jie Xiao ${ }^{1,2}$, Wende Tong1, Tao Liu¹, Bin Xiao ${ }^{1}$, Xiaofei Liu ${ }^{1}$, Youjun Feng ${ }^{1,3}$ and Quanming Zou*1

Address: ${ }^{1}$ Department of Clinical Microbiology and Immunology, College of Medical Laboratory, Third Military Medical University, Chongqing 400038, PR China, ${ }^{2}$ Department of Blood Transfusion, Chinese People's Liberation Army General Hospital of Military Region, Chengdu, 610083, PR China and ${ }^{3}$ The Department of Microbiology, University of Illinois at Urbana-Champaign (UIUC), Illinois, Urbana, 61801, USA

Email: Ying Guo - eaglegying@gmail.com; Gang Guo - guog@mail.tmmu.com.cn; Xuhu Mao - mxh95xy@mail.tmmu.com.cn; Weijun Zhang - wjzhang@mail.tmmu.com.cn; Jie Xiao - xj11mail@126.com; Wende Tong - twd8958@sina.com; Tao Liu - cedarliu@126.com; Bin Xiao - flydustyxb@yahoo.com.cn; Xiaofei Liu - liuxiaofei0401@gmail.com; Youjun Feng - fyj999@gmail.com; Quanming Zou* - qmzou@mail.tmmu.com.cn

* Corresponding author †Equal contributors

Published: 17 December 2008

BMC Microbiology 2008, 8:226 doi:10.1 186/147I-2180-8-226
Received: 25 July 2008

Accepted: 17 December 2008

This article is available from: http://www.biomedcentral.com//47|-2/80/8/226

(C) 2008 Guo et al; licensee BioMed Central Ltd.

This is an Open Access article distributed under the terms of the Creative Commons Attribution License (http://creativecommons.org/licenses/by/2.0), which permits unrestricted use, distribution, and reproduction in any medium, provided the original work is properly cited.

\begin{abstract}
Background: Iron is recognized as an important trace element, essential for most organisms including pathogenic bacteria. HugZ, a protein related to heme iron utilization, is involved in bacterial acquisition of iron from the host. We previously observed that a hugZ homologue is correlated with the adaptive colonization of Helicobacter pylori $(H$. pylori), a major gastro-enteric pathogen. However, its exact physiological role remains unclear.

Results: A gene homologous to hugZ, designated hp03/8, identified in $H$. pylori ATCC 26695, exhibits $66 \%$ similarity to $c j 16$ / 3 c of Campylobacter jejuni NCTC I I I68. Soluble $6 \times$ His fused-HugZ protein was expressed in vitro. Hemin-agrose affinity analysis indicated that the recombinant HugZ protein can bind to hemin. Absorption spectroscopy at $4 \mathrm{II} \mathrm{nm} \mathrm{further} \mathrm{revealed} \mathrm{a} \mathrm{heme:HugZ}$ binding ratio of I:I. Enzymatic assays showed that purified recombinant HugZ protein can degrade hemin into biliverdin and carbon monoxide in the presence of either ascorbic acid or NADPH and cytochrome $\mathrm{P} 450$ reductase. The biochemical and enzymatic characteristics agreed closely with those of Campylobacter jejuni CjI6/3c protein, implying that hp03/8 is a functional member of the HugZ family. A hugZ deletion mutant was obtained by homologous recombination. This mutant strain showed poor growth when hemoglobin was provided as the source of iron, partly because of its failure to utilize hemoglobin efficiently. Real-time quantitative PCR also confirmed that the expression of hugZ was regulated by iron levels.
\end{abstract}

Conclusion: These findings provide biochemical and genetic evidence that hugZ ( $h p 03 / 8$ ) encodes a heme oxygenase involved in iron release/uptake in $\mathrm{H}$. pylori. 


\section{Background}

Helicobacter pylori (H. pylori), a Gram-negative microaerophilic spiral bacterium, is known as the major pathogenic agent in a wide range of gastroenteric diseases exemplified by chronic gastritis, peptic ulcer and gastric adeno-carcinoma $[1,2]$. Increasing evidence suggests that H. pylori has adapted particularly to the niche of human stomach. Genetic diversity is widespread among the clinical isolates [3]. This polymorphism can be attributed mainly to the consequence of adaptive changes during colonization, which in turn imply that $H$. pylori has a specialized adaptation mechanism [4-6].

In our earlier study, we harvested several clinical strains of H. pylori, which initially grew weakly in Mongolian gerbils but subsequently adapted after 13 serial passages in vivo [6]. To elucidate the adaptive colonizing mechanisms of H. pylori in Mongolian gerbils further, we applied proteomic approaches to one representative $H$. pylori isolate. Fortunately, four adaptive colonization-associated proteins were identified, among which HugZ (heme iron utilization-related protein) was implicated in adaptive colonization by H. pylori for the first time [6]. However, the exact physiological role of HugZ remains elusive.

Iron is regarded as an essential trace element in living organisms, including pathogenic bacteria. It has been suggested that acquisition of iron by $H$. pylori from the host environment is required for colonization, infection and resulting disease [7-9]. Nevertheless, intracellular bacterial iron is precisely regulated and maintained at an appropriate level. Most of the free iron ion in the host is complexed with high-affinity binding proteins such as transferrin in the serum and lactoferrin on mucosal surfaces, so the level of extracellular iron available in the host is extremely low. Consequently, bacterial pathogens including $H$. pylori must have developed some mechanism to compete for the limited host iron for their survival and infection cycle [10-12].

As we know, the siderophore is a common iron acquisition apparatus/system in many pathogens; it obtains iron from transferrin or lactoferrin in the host [10,11]. Other bacteria are also capable of utilizing heme complexes as iron sources. Acquisition can be described as comprising the following steps: binding, uptake and degradation of heme [12]. Some pathogens (such as Campylobacter jejuni (C. jejuni), Vibrio cholerae and Yersinia entercolitica) have developed iron-dependent outer membrane receptors specific for heme [13-15]. Heme is transported through such receptors via a TonB-mediated gated pore mechanism $[12,15,16]$, then a periplasmic heme binding protein transports it to the cytoplasmic membrane, where a classical permease/ATPase is thought to transport it actively into the cytoplasm. Once the heme is located within the cytoplasm, a heme oxygenase protein (e.g. hemO) can utilize it. Heme oxygenase is rate-limiting in the degradation process, catalyzing the NADPH-reductase-dependent cleavage of heme to biliverdin with the release of iron and carbon monoxide $[17,18]$. In $H$. pylori, the mechanism of utilization of heme iron is not yet completely clear. Although several heme iron-repressible outer membrane proteins (IROMPs) might be involved in heme binding and/or uptake $[19,20]$ by $H$. pylori, we still do not know which component functions as the heme oxygenase. In this report, we present the functional identification of HugZ as a heme oxygenase activity in H. pylori. Our data imply that the release of iron from heme by HugZ may play a crucial role in the pathogenicity of H. pylori.

\section{Results \\ Production and evaluation of homogeneous H. pylori HugZ}

Bioinformatics analysis suggested that a hugZ homologue exists in H. pylori, which is very similar to that in C. jejuni (Fig. 1). To test its activity in iron acquisition, we prepared homogeneous $H$. pylori HugZ protein in vitro. Initially, soluble $6 \times$ His-tagged HugZ protein was expressed in a prokaryotic expression system; expression in Escherichia coli (E. coli) turned the LB medium green (data not shown), implying the presence of a reductase. This observation supports the hypothesis that catalytic turnover of Heme-HugZ triggers the accumulation of biliverdin, which is consistent with the expression profiles of prokaryotic/eukaryotic heme oxygenases [21,22]. The recombinant HugZ protein purified by a Chelating Fastflow XK1610 column $(\mathrm{CV}=18 \mathrm{ml})$ yielded $50 \mathrm{mg} /$ liter and showed about 95\% purity on 15\% SDS-PAGE (Fig. 2A), indicating high homogeneity. PMF-based sequencing showed that $H$. pylori HugZ is 251 amino acids long and shares $100 \%$ similarity to HP0318 (HugZ) protein in ATCC 26695.

To determine whether it is a functional member of the heme oxygenase family, two kinds of heme binding assay were performed. HugZ binding to hemin-agarose beads strongly indicated that it has heme-binding activity (Fig. 2B). Similarly, in vitro absorption spectroscopy suggested that HugZ is able to bind heme. As we expected, when HugZ was mixed with hemin, the spectrum of the complex showed a typical spectrographic curve with a prominent Soret peak at $411 \mathrm{~nm}$, and a shoulder at $540 \mathrm{~nm}$ and a smaller peak at $580 \mathrm{~nm}$, corresponding to the $\beta$ - and $\alpha$ porphyrin bands of the heme-HugZ complex respectively (Fig. 3A). To quantify heme binding, HugZ solution (20 $\mu \mathrm{M}$ ) was titrated with increasing amounts of hemin (Fig. 3 ). The increase in absorption leveled off at approximately $20 \mu \mathrm{M}$ heme, showing a 1:1 stoichiometry of heme to HugZ (Fig. 3B). 


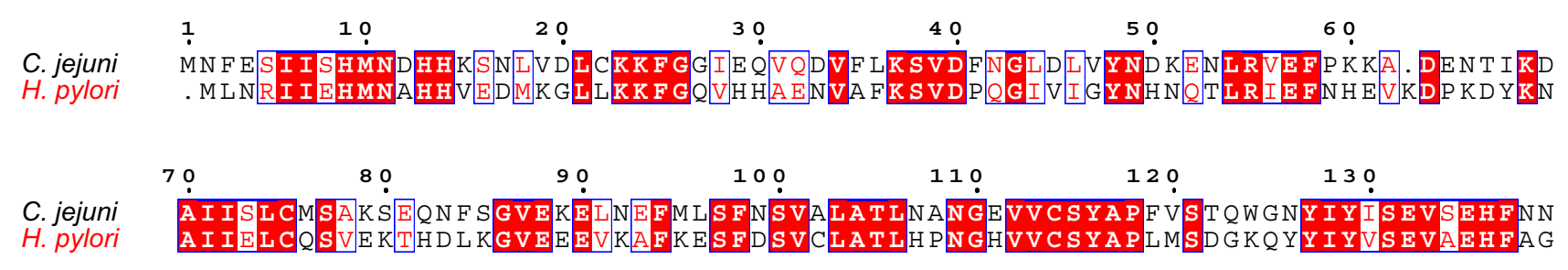

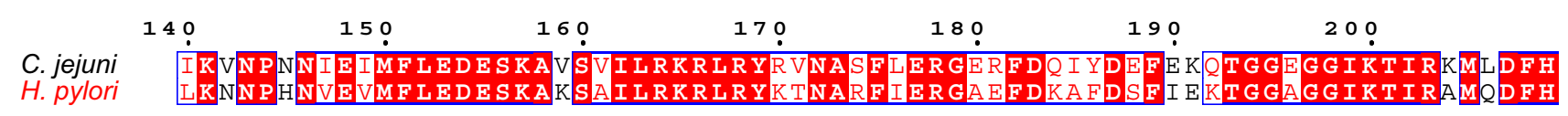

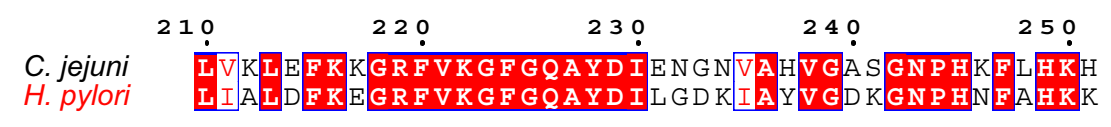

Figure I

Amino acid sequence alignment of the $C$. jejuni heme oxygenase (Cj I 6 I 3c) with $H$. pylori HugZ. The alignment was performed using the WebESPript 2.2 program on the Institut de Biologie et Chimie des Protéines website.

\section{HugZ catalyzes the degradation of heme}

It has been suggested that some heme binding proteins can degrade heme by so-called coupled oxidation, a nonenzymatic mechanism [14]. Coupled oxidation involves the generation of peroxide by the heme protein and is prevented if catalase is present. Heme oxygenases catalyze the opening of the heme macro-cycle in the presence of an electron donor. Purified heme oxygenase has been shown not to release the product biliverdin readily in the absence of biliverdin reductase [21]. Thus, most studies involve single turnover assays, as was done here. In addition, the in vivo electron donor for bacterial heme oxygenases is not known, but ascorbate or NADPH-cytochrome P450 reductase may be used for catalysis by the pure enzyme $[21,23]$. In the first experiment, heme degradation catalyzed by HugZ was measured spectrophotometrically using human NADPH-CPR as the electron donor (Fig. 4A). NADPH was added to the reaction mixture in $10 \mu \mathrm{M}$ increments and the mixture was scanned from 350 to 800 $\mathrm{nm}$ after each addition. The Soret band decreased successively after addition of NADPH. Finally, the HugZ substrate-hemin was exhausted and the NADPH was not oxidized completely, so there was absorption at $340 \mathrm{~nm}$ due to NADPH. Heme degradation did not occur if HugZ, NADPH or CPR was omitted from the reaction mixture (not shown).

In the second experiment, the HugZ-dependent disappearance of heme was measured using $20 \mathrm{mM}$ ascorbate as the reductant (Fig. 4B). Heme was degraded more rapidly with ascorbate than with human NADPH-CPR, and most of the decrease was complete by 20 min after the ascorbate was added. No degradation of heme was observed in the absence of HugZ or ascorbate (not shown). Collectively, these findings showed that HugZ catalyzes the enzymatic degradation of heme.

\section{Biliverdin and $\mathrm{CO}$ produced by HugZ-catalyzed heme degradation}

Biliverdin is the final product of heme degradation by heme oxygenases. When heme was degraded by HugZ, a broad absorbance peak in the 660-nm region became prominent, implying is the presence of biliverdin. To determine the kind of biliverdin formed, we subjected this product to HPLC analysis. HPLC chromatography of all four possible biliverdin isomers is shown for comparison (Fig. 5). The HPLC profiles of the products formed during HugZ-catalyzed heme degradation with ascorbate and NADPH gave a retention time and absorption spectrum identical to that of biliverdin IX $\delta$.

The much higher affinity of myoglobin for $\mathrm{CO}$ than for oxygen allows the $\mathrm{CO}$ produced by oxidative cleavage of the heme to be detected [21]. Difference absorption spectroscopy in the presence of myoglobin confirmed $\mathrm{CO}$ as a product of the oxidative cleavage of heme by HugZ. The myoglobin absorption spectrum was recorded at 2-min intervals in order to monitor the characteristic spectral changes of a myoglobin-CO complex (Fig. 6). The transition of ferrous-dioxygen myoglobin to the ferrous-CO myoglobin complex was associated with a shift in the Soret band from 411 to $421 \mathrm{~nm}$ as well as the appearance of bands at 540 and $580 \mathrm{~nm}$. Control reactions in the absence of the heme-HugZ complex showed no shift in the Soret band. The complete conversion indicated that 


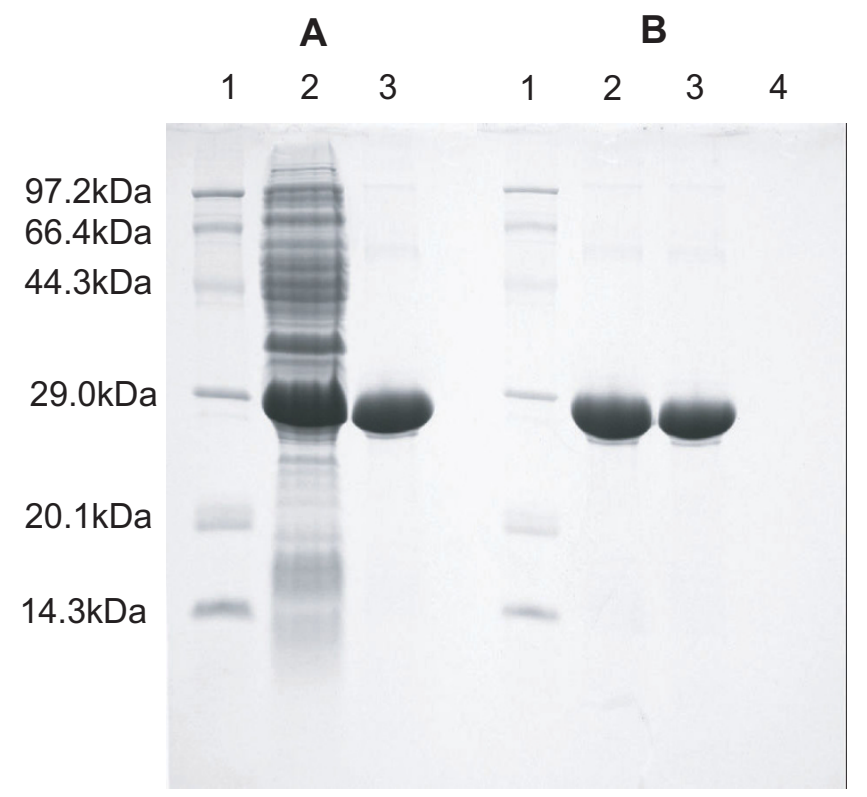

Figure 2

SDS-PAGE of the purified recombinant HugZ protein and binding of HugZ to hemin-agarose. (A) lane $I$, molecular mass markers; lane 2, pET22b-hugZIE. coli BL2I (DE3); lane 3, purified $6 \times$ His-HugZ; (B): lane I, molecular mass markers;lane 2, purified $6 \times$ His-HugZ;lane 3, proteins that bound to the hemin-agarose; lane 4, proteins that bound to the hemin-agarose after preincubation with 10 $\mathrm{nmol}$ hemin. The data are representative of triplicate independent experiments.

carbon monoxide as well as biliverdin was generated as a product of oxidative heme cleavage in $\mathrm{H}$. pylori.

\section{HugZ is a cytoplasmic protein}

To determine the cellular location of HugZ, Immunoelectron microscopy (IEM) was performed. Frozen sectioned samples of $H$. pylori 26695 strains were treated with antiHugZ antibodies and gold-labeled secondary antibodies. Analysis of the positions of the gold particles (Fig. 7B) revealed that HugZ was predominantly located in the cytoplasm in $H$. pylori cells.

\section{The hugZ mutant fails to utilize heme iron for normal growth}

In order to elucidate the role of HugZ, the mutant $\Delta h u g Z$ was obtained from more than $100 \mathrm{H}$. pylori transformants. The correct genotype of $\Delta h u g Z$ was systemically confirmed by PCR (Fig. 8A \& 8B), RT-PCR (Fig. 8C) and direct DNA sequencing (data not shown).

The hugZ deletion mutant ( $\triangle$ hugZ) grew normally in liquid BBF and on BBF blood agar plates, indicating that HugZ is not required for bacterial growth under iron-
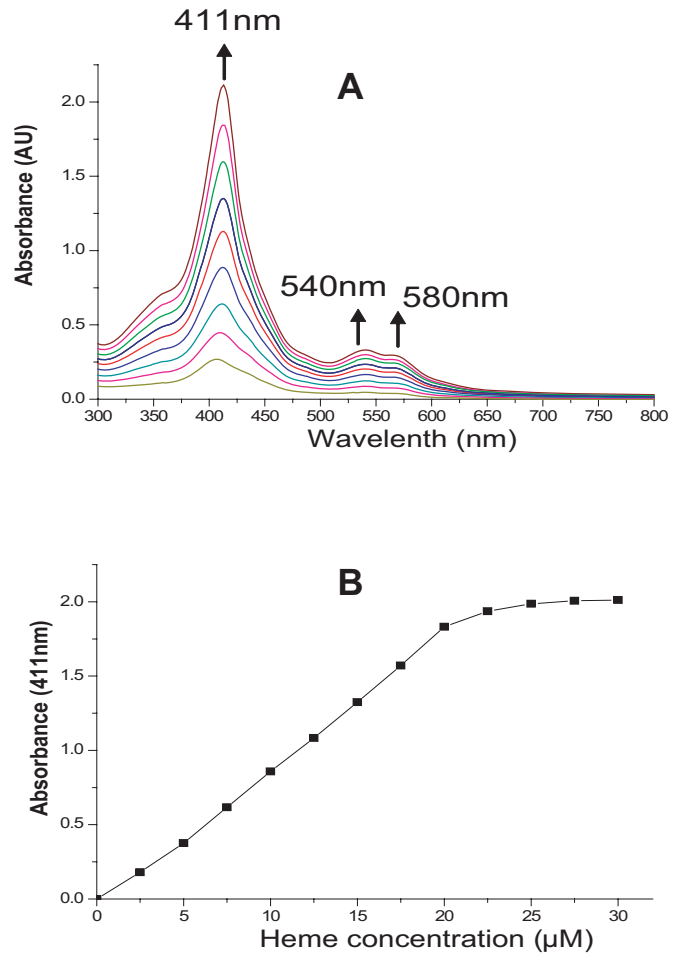

Figure 3

Absorption spectroscopy of hemin binding to recombinant HugZ. (A) Absoption profile of hemin at various concentrations. Hemin $(2.5 \mathrm{mM}$ in $20 \mathrm{mM} \mathrm{NaOH})$ was titrated in $2.5 \mu \mathrm{M}$ increments $(0,2.5,5,7.5,10,12.5,15$, 17.5, 20, 22.5, 25, 27.5, $30 \mu \mathrm{M}$ ) against $20 \mu \mathrm{M} 6 \times$ His-HugZ. Absorbance changes are indicated by the position and direction of the arrows. (B) Hemin: $6 \times$ His-HugZ binding stoichiometry and affinity. Values were plotted as change in absorbance at $4 \mathrm{II} \mathrm{nm}$ against hemin concentration. The data are representative of triplicate independent spectrophotometric analyses.

replete conditions. Subsequently, we tested its growth in the presence of different iron sources. $\Delta h u g Z$ strains showed poor growth in iron-restricted conditions while the wild type grew well (Fig. 9). These data suggest that the hugZ mutant cannot utilize heme iron for normal growth.

\section{Regulation of hugZ expression by iron}

Merrell et al. reported that hugZ ( $h p 0318$ ) was one of the genes induced by iron starvation [24]. To test whether hugZ is regulated by iron, real-time quantitative PCR was performed. The effects of different iron levels on hugZ transcription varied (Fig. 10). Transcription was suppressed by $\mathrm{FeCl}_{3}$ (compared to $\mathrm{BBF}$, the change fold ratio was $0.410 \pm 0.056(p<0.01$, Student's $t$-test $))$ and stimulated under iron-restricted conditions (compared to BBF, the change fold ratio was $3.90 \pm 0.010(p<0.01$, Student's 

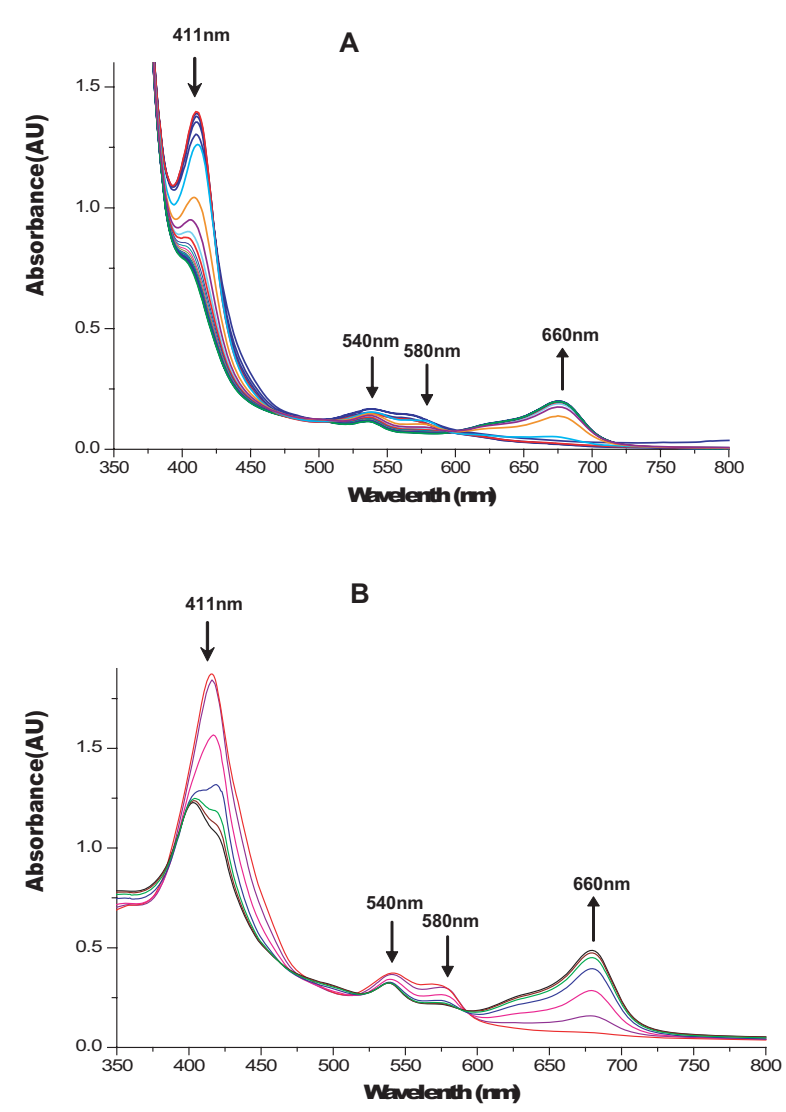

Figure 4

Degradation of the heme:HugZ complex in the presence of NADPH-cytochrome $\mathbf{P 4 5 0}$ reductase and ascorbic acid. (A) Degradation of hemin using recombinant human NADPH-cytochrome $\mathrm{P} 450$ reductase as the reductant. Arrows indicate the positions and directions of absorbance change with time. (B) Degradation of hemin using ascorbate as the reductant. The arrow indicates changes in absorption with time. The data are representative of triplicate independent spectrophotometric analyses.

$t$-test)). These results indicated that hugZ (hp0318) is down-regulated by iron.

\section{Discussion}

A wide array of metal ions including iron, copper and nickel are known to be closely related to $H$. pylori colonization and infection $[25,26]$. Iron metabolism-related proteins play important roles in $H$. pylori infections. However, the iron-specific metabolic mechanism in $H$. pylori is still not well understood. Bacteria require iron to complete their life cycles and in particular for growth and infection. The limited availability of extra-cellular iron in the host, which is partly due to iron insolubility, restricts
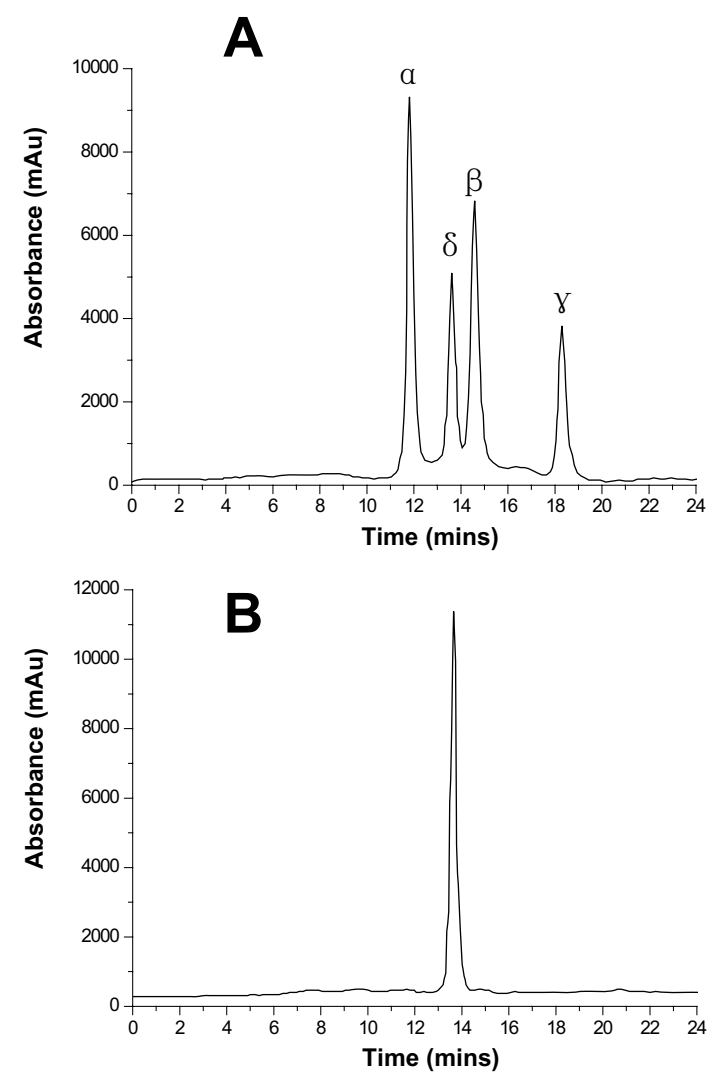

\section{Figure 5}

HPLC detection of the product of the HugZ reaction with NADPH cytochrome $\mathbf{P 4 5 0}$ reductase. (A): HPLC chromatogram of a mixture of all four biliverdin isomers as standards. (B): Spectroscopy and HPLC product analysis both show that a product of heme degradation by HugZ is the biliverdin IX $\delta$ isomer. The data are representative of three independent HPLC runs.

microbial growth greatly, so iron acquisition seems to be crucial for the survival of pathogens. Actually, it has been suggested that bacteria evolve sophisticated systems to compete for iron with their hosts. In general, heme is an important iron source in hosts and it can be utilized by most pathogens. Heme is degraded by heme oxygenase in the bacterial cytoplasm, releasing the iron.

Heme oxygenase is the rate-limiting enzyme in heme degradation; it catalyzes reduction system-dependent cleavage of heme to biliverdin with the release of iron and carbon monoxide. It is reasonable to suppose that bacterial heme oxygenase releases the iron from heme for subsequent use by the invading pathogen [18]. Heme oxygenases are widespread among pathogenic bacteria such as C. jejuni and Y. pestis and play key roles in the growth and colonization of those pathogens $[13,27]$. 


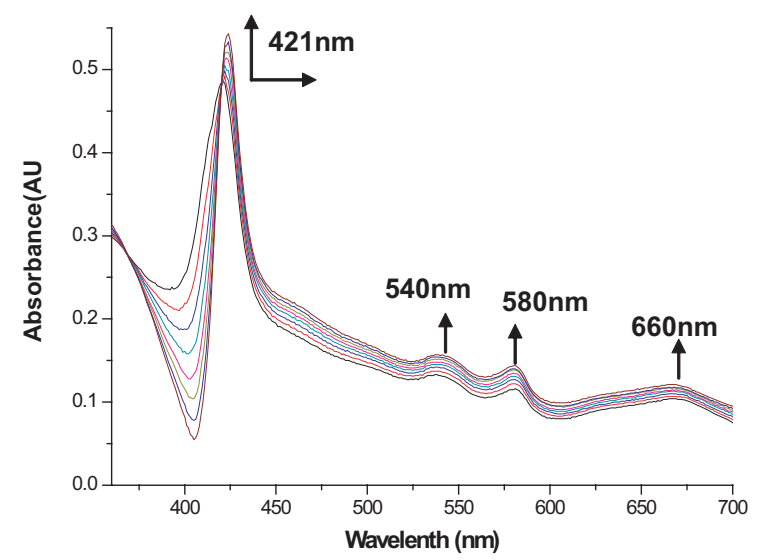

Figure 6

Difference absorption spectra of the heme-HugZ and NADPH cytochrome $\mathbf{P 4 5 0}$ reductase reaction in the presence of myoglobin. The reference and sample cuvettes contained the hemin-HugZ complex $(20 \mu \mathrm{M})$, recombinant human NADPH reductase $(100 \mu \mathrm{g})$ and NADPH (I00 $\mu \mathrm{M})$. The reaction was blanked immediately after the addition of NADPH, and myoglobin (I25 $\mu \mathrm{M})$ was added to the sample cuvette. The shift in the Soret band from $4 \mathrm{II}$ to $42 \mathrm{I} \mathrm{nm}$ was monitored at I-min intervals for 10 $\min$. The data are representative of three independent experiments.

Heme oxygenase mutants of Corynebacterium diphtheriae and Neisseria meningitidis were unable to utilize heme or hemoglobin as an iron source $[28,29]$. Similarly, it has been suggested that heme oxygenase (Cj1613c) is necessary for growth in C. jejuni [13]. For H. pylori, the role of heme degradation in iron metabolism is relatively obscure. In this study, we identified a heme oxygenase called HugZ that is responsible for heme iron utilization in H. pylori. The heme oxygenase activity of HugZ was confirmed by the appearance of characteristic spectral changes following addition of ascorbic acid or a NADPHCPR system as electron donor. HugZ binds to hemin in vitro at $1: 1$ and produces absorbance bands at 411, 540 and $580 \mathrm{~nm}$, which are similar to those reported for other heme oxygenases such as ChuS [30] and Cj1613c [13]. The formation of a broad absorbance band at 395 and $660 \mathrm{~nm}$ suggests that the end product of heme degradation is iron-free biliverdin rather than ferric biliverdin [28].

We demonstrated that the products of hugZ cleave heme to carbon monoxide and biliverdin IX $\delta$, which shows that the $\delta$-meso carbon bridge position in the heme precursor is eliminated by HugZ. As with various eukaryotic and prokaryotic heme oxygenases, overexpression of HugZ in

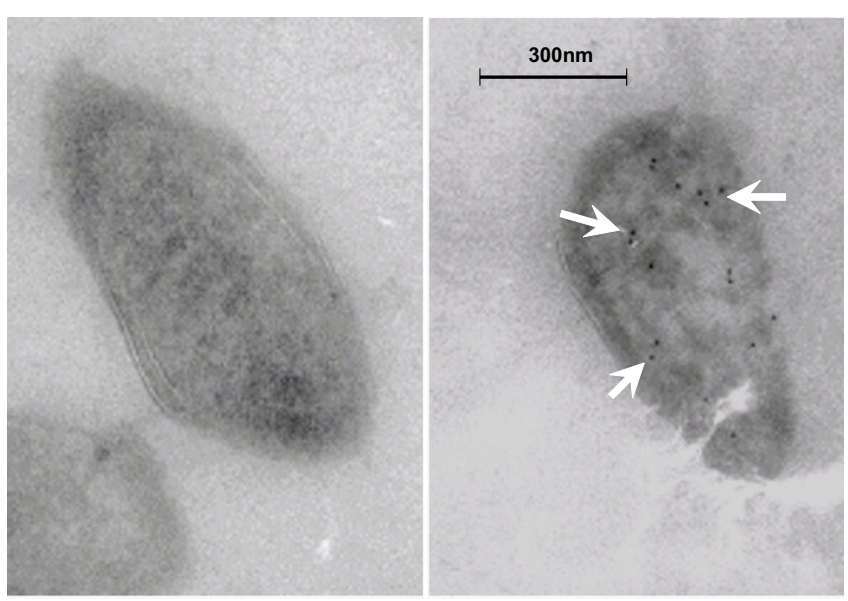

A $\quad$ B

Figure 7

IEM analysis of HugZ location in H. pylori 26695. Representative immuno-electron micrographs of frozen thin-sectioned specimens are shown. Labeling in the $H$. pylori cytoplasm (B) was strong in comparison to the negative control (A). The cell wall shows different widths depending on the plane of section. Scale bar, $300 \mathrm{~nm}$.

E. coli can make the culture medium green owing to the accumulation of biliverdin. It is presumed that during the expression of those exogenous heme oxygenases in E. coli, reducing systems in the bacterium support the catalytic turnover of heme $[21,22]$. Furthermore, several reduction systems including human CPR and ascorbic acid can support biliverdin production by purified recombinant HugZ in vitro. These results suggest that $H$. pylori probably has the same reduction partners for HugZ-heme oxygenase activity.

Further experiments showed that the H. pylori hugZ mutant exhibited poor growth, though wild type strains grew well when heme was added to iron-restricted BBF, indicating that the hugZ mutant cannot effectively utilize hemoglobin as a heme iron source, which confirmed that HugZ is a heme oxygenase.

In mammals, the primary function of heme oxygenases is to maintain iron homeostasis, whereas prokaryotic heme oxygenases help bacteria to take in iron from heme. Most bacterial heme oxygenases are regulated by the ferric uptake repressor (Fur). Fur requires iron to bind to target DNA sequences (Fur-boxes) and controls the expression of iron-regulated genes [18]. Merrell and Gancz et al. used a Microarray to analyze the expression of iron-regulated genes in H. pylori and reported that $h p 0318$ ( $h u g Z)$ is one of the genes induced by iron starvation; they presumed that a hypothetical Fur box located before $h p 0321$ controls 

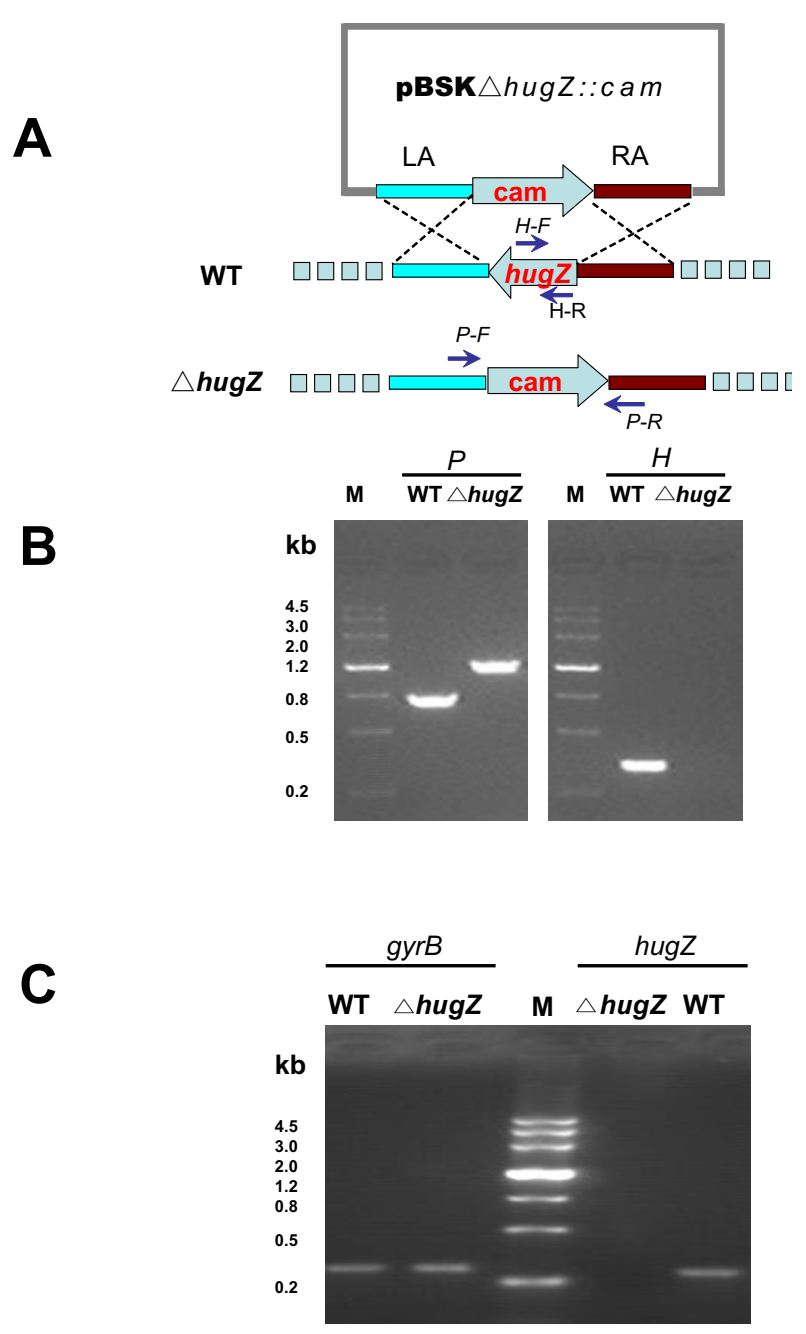

\section{Figure 8}

Identification of hugZ, the isogenic mutant of hugZ in H. pylori 26695. (A) Cartoon description of hugZ knockout from the $H$. pylori 26695 chromosome. pBSK $\Delta$ hugZ::cam is the recombinant vector constructed specifically to inactivate hugZ. hugZLA and hugZRA respectively indicate the left- and right- border of hugZ. A pair of specific primers (P-F \&P-R) located adjacent to hug $Z$ on both sides are indicated with blue arrows and used for PCR-detection of hugZ in the $H$. pylori 26695 genome. WT, wild type $H$. pylori 26695; $\Delta$ hugZ, an isogenic mutant of gene hugZ. (B) Multiple-PCR analysis of $\Delta$ hugZ. The PCR products were separated by electrophoresis on a $1.0 \%$ agarose gel stained with ethidium bromide (EB). $P \& H$, the $P C R$ product amplified with the $P-F \& P-R$ and $H-F$ $\& H-R$ primers for hugZ. $\Delta$ hugZ has been replaced by $C a m^{R}$ without affecting either boundary sequence (not shown). (C) RT-PCR analysis of $\triangle$ hugZ using hugZI \& hugZ2 and gyrBl \& gyrB2 primers. RT-PCR products of hugZ and gyrB were separated by electrophoresis on a $2.0 \%$ agarose gel.

hp0318 expression [24,31]. Our real-time quantitative RT PCR results also support that the view that hugZ ( $h p 0318$ ) is down-regulated by iron. However, further studies are needed to determine whether the presumed Fur box controls the transcription of $h u g Z$.

\section{Conclusion}

Taken together, these findings confirm that $H$. pylori HP0318 (HugZ) is a heme oxygenase. Our data imply that HugZ may play a crucial role in the acquisition of heme iron by H. pylori.

\section{Methods \\ Bacterial strains and growth conditions}

H. pylori strain ATCC 26695 was cultivated in liquid Brucella Broth with $10 \%$ fetal bovine serum (BBF) and a mixture of antibiotics $(10 \mu \mathrm{g} / \mathrm{ml}$ vancomycin, $5 \mu \mathrm{g} / \mathrm{ml}$ trimethoprim, $6 \mu \mathrm{g} / \mathrm{ml}$ nalidixic acid and $5 \mu \mathrm{g} / \mathrm{ml}$ amphotericin B). The solid medium consisted of the aforementioned ingredients with 5\% rabbit blood and 1.5\% agar at $37^{\circ} \mathrm{C}$ under microaerobic conditions $\left(10 \% \mathrm{CO}_{2}, 85 \% \mathrm{~N}_{2}\right.$, $5 \% \mathrm{O}_{2}$ ) [32]. Iron-replete conditions were achieved by adding $\mathrm{FeCl}_{3}$ to a final concentration of $50 \mu \mathrm{M}$. Ironrestricted conditions were achieved by adding the iron chelator desferrioxamine mesylate (Desferal) to a final concentration of $75 \mu \mathrm{M}$ [19]. H. pylori strains were grown in the presence of heme as the sole iron source at a final concentration of $12.5 \mu \mathrm{M}$ hemoglobin in iron-restricted BBF. The strains were initially cultured on BBF blood agar plates overnight, harvested in a suitable volume of BBF, and used to inoculate $5 \mathrm{ml} \mathrm{BBF}$ to an optical density at $600 \mathrm{~nm}\left(\mathrm{OD}_{600}\right)$ of 0.05 . The cultures were incubated microaerobically with shaking, and the optical density was monitored at regular time intervals.E. coli strains DH5 $\alpha$ and BL21 (DE3) were used as cloning host and expression host, respectively. Antibiotic selection was achieved when necessary by addition of ampicillin (100 $\mu \mathrm{g} / \mathrm{ml})$ or chloramphenicol $(10 \mu \mathrm{g} / \mathrm{ml})$.

\section{Construction of hugZ knockout mutant}

$h u g Z$ was activated by allelic replacement with a constitutively-expressed chloramphenicol resistance $\left(\mathrm{Cam}^{\mathrm{R}}\right)$ cassette. First, the DNA sequences flanking hugZ, including 1000 base pairs upstream and 1000 base pairs downstream, were amplified from the chromosomal DNA of $H$. pylori 26695 using PCR with two pairs of specific primers (LA-F1/LA-R1 and RA-F1/RA-R1) carrying SacI/XbaI and SmaI/SalI restriction enzyme sites, respectively (Table 1). After digestion with the corresponding restriction enzymes, the DNA fragments were cloned directionally into a pBluescript II SK(-) vector. The $\mathrm{Cam}^{\mathrm{R}}$ gene cassette (from phel2 [33]) was then inserted at the XbaI/SmaI sites to generate the hugZ knockout vector pBSK $\Delta h u g Z:: \mathrm{cam}^{R}$. To obtain the isogenic mutant $\Delta h u g Z$, the plasmid pBSK$\Delta$ hugZ:: $\mathrm{cam}^{R}$ was electrotransformed into H. pylori 26695, where the $\mathrm{Cam}^{\mathrm{R}}$ marked mutation was introduced into the genome by homologous recombination, resulting in the hugZ:: $\mathrm{cam}^{R}$ mutant strain. PCR was used to examine all the $\mathrm{Cam}^{\mathrm{R}}$ transformants with a series of specific primers. 


\section{A: Iron replete}

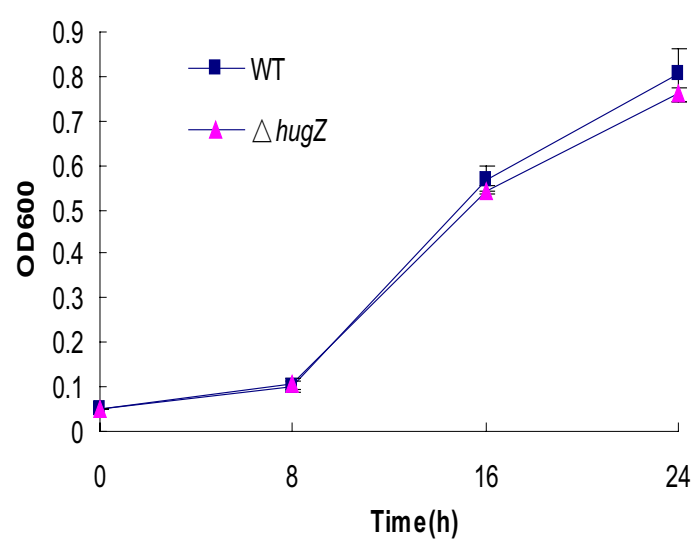

\section{B: Iron restric ted}

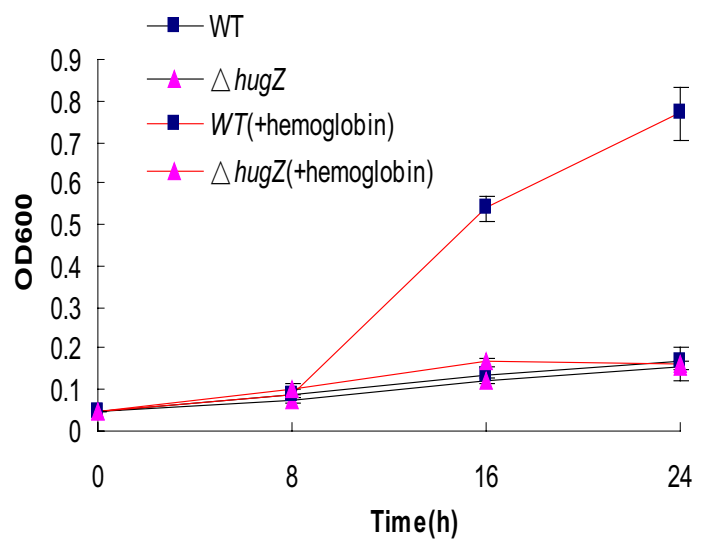

\section{Figure 9}

Growth of hugZ mutants. Samples were tested in triplicate, and the data plotted are the means of three independent experiments together with the sample error. Symbols: A: H. pylori 26695 WT and $\triangle$ hugZ strains grown in BBF supplemented with 50 $\mu \mathrm{M} \mathrm{FeCl}_{3}$ (iron-replete); B: WT and $\Delta$ hugZ strains grown in BBF plus $75 \mu \mathrm{M}$ desferal (iron-restricted) with or without $12.5 \mu \mathrm{M}$ $\mathrm{Hb}$ supplement. The optical density of the bacteria was monitored at $600 \mathrm{~nm}$. The key to symbols is shown in figure. Error bars indicate standard deviation from three replicate cultures. $\mathrm{Hb}$, hemoglobin.

\section{Overexpression and purification of recombinant HugZ and preparation of HugZ antiserum}

After the amino acid sequence of HugZ (HP0318 located in Helicobacter pylori 26695) was aligned with that of Cj1613c of C. jejuni NCTC 11168 [13,32], it was recognized as a candidate/functional member related to iron acquisition. To test this bioinformatics-based hypothesis, the full length $753 \mathrm{bp} h u g Z$ was amplified with primers $H_{1}$ (with NdeI site) and $\mathrm{H}_{2}$ (with XhoI site) (Table 1), using genomic $H$. pylori ATCC 26695 DNA as template. The PCR conditions were initial denaturation for $10 \mathrm{~min}\left(95^{\circ} \mathrm{C}\right)$ followed by 35 cycles of amplification $\left(40 \mathrm{~s}\right.$ at $95^{\circ} \mathrm{C}, 30 \mathrm{~s}$ at $53^{\circ} \mathrm{C}$ and $1 \mathrm{~min}$ at $72^{\circ} \mathrm{C}$ ) and a final extension for 10 min at $72^{\circ} \mathrm{C}$, using a gene cycler (BIORAD). The PCR product was cloned into the pMD-18T vector (Takara), generating pMD-18T-hugZ, and directly subcloned into pET-22b (+) (Novagen) via Nde I and Xho I restriction sites, resulting in the recombinant expression plasmid pET-22b-hugZ. Finally, the positive clones were further confirmed by direct DNA sequencing. For the expression of HugZ protein, an overnight culture of strain BL21 (pET22b-hugZ) was diluted 1:100 into $2000 \mathrm{ml}$ of LB medium; $0.5 \mathrm{mM}$ IPTG (isopropyl $\beta$-D-thio $\beta$-D-galactopyranoside; Sigma) was added when the $\mathrm{OD}_{600}$ reached 0.6 and the culture was maintained for $12 \mathrm{~h}$ at $16^{\circ} \mathrm{C}$ with shaking. Cells were harvested by centrifugation and resuspended in $200 \mathrm{ml}$ of $20 \mathrm{mM}$ Tris- $\mathrm{HCl} 0.5 \mathrm{M} \mathrm{NaCl}(\mathrm{pH}$
7.8). After homogenization 5 times in an APV1000 High Pressure Homogenizer (Denmark) at 750 bar on ice, the sample was centrifuged at $12,500 \times \mathrm{g}$ for $30 \mathrm{~min}$ and filtered through a $0.45-\mu \mathrm{m}$-pore-size filter (Sartorius). The recombinant HugZ was purified using the AKTÄ Explorer100 system with a Chelating Fastflow XK1620 column $(\mathrm{CV}=18 \mathrm{ml})(\mathrm{GE})$ in accordance with the manufacturer's standard protocol. Protein purity was determined by SDS-PAGE. Also, Peptide Mass Fingerprint (PMF) analysis was used to identify the HugZ protein (Beijing Genomics Institute). The purified HugZ protein was concentrated and dialyzed three times (Vivaspin 20 centrifugal concentrators, $10 \mathrm{kDa}$ molecular weight cut off, Sartorius) against $20 \mathrm{mM}$ Tris- $\mathrm{HCl}(\mathrm{pH} 7.8)$ at $4{ }^{\circ} \mathrm{C}$ and quantified by the Lowry Method $(600 \mu \mathrm{g} / \mathrm{ml})$. The purified protein was used to prepare anti-HugZ antiserum in rabbits in accordance with standard protocols.

\section{Immunoelectron microscopy (IEM)}

IEM was performed as described by Michie et al. [34]. Wild type $H$. pylori 26695 cells were grown in BBF at $37^{\circ} \mathrm{C}$ overnight, fixed with $10 \%$ glutaraldehyde and washed before dehydration at $4{ }^{\circ} \mathrm{C}$ in $80 \%$ ethanol. The cells were frozen in liquid nitrogen for use. For immunolabeling, frozen ultrathin sections were collected on Formvarcoated gold slot grids. Sections were treated with blocking buffer then incubated for $4 \mathrm{~h}$ at room temperature with 


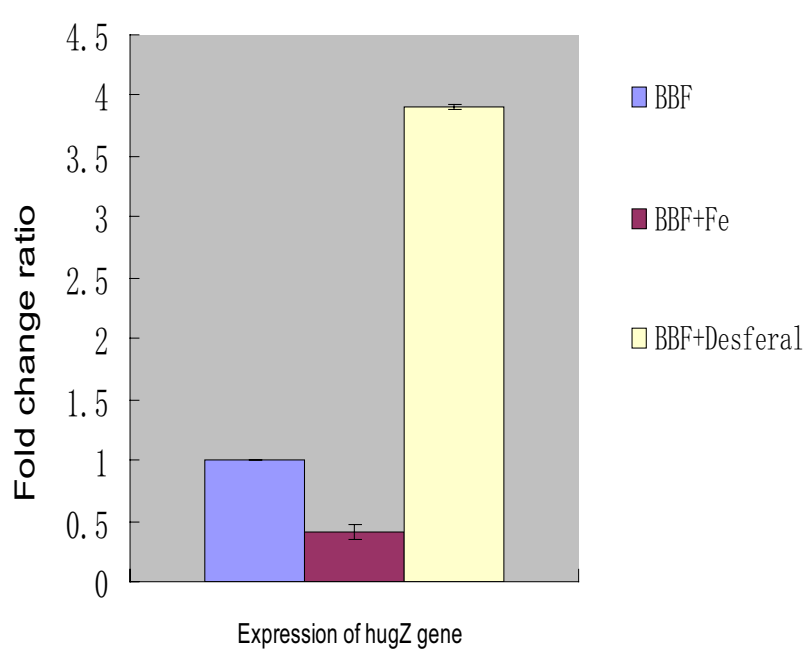

Figure 10

Comparison of the levels of hugZ expression under different iron level conditions, detected by real-time quantitative RT-PCR. The results are based on the ratio hugZ mRNA amplification/gyrB mRNA amplification, which are presented as the fold induction of mRNA expression relative to the amount present in BBF. Real-time PCR was conducted in duplicate for each sample and the mean value was calculated. Expression values were calculated from three biological replicates. (BBF: Brucella Broth with $10 \%$ fetal bovine serum; $\mathrm{BBF}+\mathrm{Fe}$ : $\mathrm{BBF}$ plus $50 \mu \mathrm{M} \mathrm{FeCl}_{3}$; $\mathrm{BBF}+$ Desferal: $\mathrm{BBF}$ plus $75 \mu \mathrm{M}$ Desferal).

affinity-purified anti-HugZ rabbit antiserum diluted 1:2000 in BB or with BB alone (as a negative control). The grids were washed six times with wash buffer (PBS 0.05\% Tween 20), blocked with 5\% normal goat serum and incubated in goat anti-rabbit 15-nm gold diluted 1:100 in BB plus 5\% goat serum. The grids were washed twice with wash buffer, twice with PBS and twice with water before staining in saturated aqueous uranyl acetate for $20 \mathrm{~min}$. Sections were viewed under a Philips Tecnai 10 transmission electron microscope.

\section{Binding of HugZ to hemin}

Two independent assays, which involved hemin-agarose beads and spectrophotometry, were utilized to test the binding activity of HugZ. The hemin agarose-based assay was performed as described by Lee [35]. In brief, $100 \mu \mathrm{l}$ of hemin-agarose (Sigma-Aldrich) was washed thrice in 10 $\mathrm{ml} 0.5 \mathrm{M} \mathrm{NaCl}-20 \mathrm{mM}$ Tris- $\mathrm{HCl}$ (pH 7.8), then incubated with purified HugZ $(20 \mu \mathrm{g})$ with or without $10 \mathrm{nmol}$ hemin for $30 \mathrm{~min}$. After the removal of contaminants, the bound protein was analyzed by $15 \%$ SDS-PAGE. The absorption spectroscopy assay was carried out as described by Wilks et al. [21]. One milliliter of $20 \mu \mathrm{M}$ HugZ (in $20 \mathrm{mM}$ Tris- $\mathrm{HCl}(\mathrm{pH} 7.8)$ ) was applied at $25^{\circ} \mathrm{C}$. Hemin ( $2.5 \mathrm{mM}$ in $20 \mathrm{mM} \mathrm{NaOH}$ ) was titrated against the HugZ in $2.5 \mu \mathrm{M}$ increments and the absorbance spectrum between 300 and $800 \mathrm{~nm}$ was recorded on a TU-1901 spectrophotometer (Pgeneral, China). The absorption at $411 \mathrm{~nm}$ was plotted against the heme concentration.

\section{Determination of hugZ heme oxygenase activity}

Heme-HugZ complex was prepared at a hemin:protein ratio of 2:1 and excess heme was removed by filtration through a Sephadex G-25 column. Degradation of HugZbound hemin to biliverdin was mediated by two electrondonor systems (ascorbic acid and NADPH-CPR). Ascorbic acid was added to a final concentration of $20 \mathrm{mM}$. In the NADPH-CPR system, heme-HugZ protein $(20 \mu \mathrm{M})$ was added to $100 \mu \mathrm{g}$ of recombinant human NADPH-cytochrome $\mathrm{P} 450$ reductase (CPR). The reaction was initiated by adding NADPH to $100 \mu \mathrm{M}$ and spectra were recorded from $350 \mathrm{~nm}$ to $800 \mathrm{~nm}$ every $2 \mathrm{~min}$ for $1 \mathrm{~h}$. In order to avoid the involvement of non-enzymatic $\mathrm{H}_{2} \mathrm{O}_{2}$-mediated conversion of heme to biliverdin, $2 \mu \mathrm{M}$ catalase (bovine

Table I: Oligonucleotide primers used in this study

\begin{tabular}{|c|c|c|c|}
\hline Primer & Sequence $\left(5^{\prime} \rightarrow 3^{\prime}\right)$ & Characteristics & Functions (genes) \\
\hline $\mathrm{HI}$ & CGCGCATATGCTTAATCGTATC & Ndel & To amplify gene hugZ \\
\hline $\mathrm{H} 2$ & GGCCTCGAGTTTCTTGTGAGCG & Xhol & \\
\hline LA-FI & CGAGCTCCCATTACTACTGCTACTACTA & Sacl & To amplify left arm of gene hugZ (LA) \\
\hline$L A-R I$ & GCTCTAGAGCACCGCTCATAAGGGGCAA & Xbal & \\
\hline RA-FI & TCCCCCGGGGGAAATATTCTCCTTAGTT & Smal & To amplify right arm of gene hugZ (RA) \\
\hline$R A-R I$ & ACGTCGACGCAATGCTTTTAGAAATTAA & Sall & \\
\hline$H-F$ & TCGCTAAACAAACAGAATCAA & l & To detect hugZ \\
\hline$H-R$ & ATGTGTTCTATGATACGATTAAGCAT & l & \\
\hline$P-F$ & GCTCTAGAGCTTGCCCCTTATGAGCGGT & I & To detect hugZ or cam \\
\hline$P-R$ & CTTATTTTTTGAAACTAAGGAGAATATT & l & \\
\hline hugZ-I & TTGGGCAAGTCCATCACG & $245 \mathrm{bp}$ & RT-PCR/Real-time RT-PCR Evaluation of hugZ \\
\hline hugZ-2 & GGTCGCTAAACAAACAGAA & & \\
\hline gyrB-l & CGCTAAAGAAAGTGGCACGA & $267 \mathrm{bp}$ & RT-PCR/Real-time RT-PCR Evaluation of gyrB (normalizer) \\
\hline gyrB-2 & TGCGCGTTTCTTCATCCAT & & \\
\hline
\end{tabular}

The underlined sequences are the restriction sites. /: Absence of restriction endoenzyme sites. 
liver, Sigma-Aldrich) was added to the reaction systems [13]. Finally, two heme-HugZ reaction products, biliverdin and carbon monoxide (CO), were determined. First, HPLC was used to detect biliverdin [21]. Second, to determine CO, recombinant human NADPH-CPR $(100 \mu \mathrm{g})$ and NADPH $(100 \mu \mathrm{M})$ were placed in both the reference and reaction cuvettes in a final volume of $3 \mathrm{ml}$ and blanked immediately. Then $150 \mu \mathrm{l}$ of myoglobin (125 $\mu \mathrm{M})$ (Sigma-Aldrich) was added to the reaction cuvette and the same volume of buffer to the reference cuvette. Spectra were recorded every 2 min between 400 and 700 $\mathrm{nm}$ for up to $1 \mathrm{~h} \mathrm{[13].}$

\section{Transcriptional analysis of hugZ by real-time RT-PCR} H. pylori RNA was isolated using TRIzol reagent (Gibco/ $\mathrm{BRL}$ ). The RNA concentration was quantified by the $\mathrm{OD}_{260}$, and RNA integrity was verified by visualization on a $2 \%$ agarose gel. Real-time quantitative PCR was performed as described by Feng et al. with a minor modification [36]. Briefly, hugZ-specific primers (hugZ1 and hugZ2) (Table 1) and SYBR Green PCR master mix (ABI) were used. Real-time PCR was performed using a RotorGene 6000 real-time PCR system (Corbett Life Science, Australia). Known concentrations of $H$. pylori 26695 genomic DNA were used to construct a gene-specific standard curve so that the concentration of template in each reaction could be determined. The gene encoding DNA gyrase subunit B, GyrB (HP0501) [37], was used to normalize all reactions. Melting curve analysis confirmed that all PCRs amplified a single product.

\section{Authors' contributions}

QZ, GG and XM conceived and designed the experiments. YG, GG, WZ, JX, TL, BX and XL performed the experiments. YG, YF, GG and XM analyzed the data. GG, WT and YF contributed reagents/materials/analysis tools. YG, YF and GG wrote the paper.

\section{Acknowledgements}

We thank Dr. Hong Zhou for the gift of recombinant human p450 CPR, Dr. Yonghong Zhu for detecting the biliverdin by HPLC and Drs. JunYang \& PingYang for performing the IEM. This work was supported by the National Natural Science Foundation of China (30400019).

\section{References}

I. Graham DY, Lew GM, Klein PD, Evans DG, Evans DJ Jr, Saeed ZA, Malaty HM: Effect of treatment of Helicobacter pylori infection on the long-term recurrence of gastric or duodenal ulcer. A randomized, controlled study. Ann Intern Med 1992, I | 6(9):705-708

2. Wotherspoon AC: Helicobacter pylori infection and gastric lymphoma. Br Med Bull I998, 54(I):79-85.

3. Gottke MU, Fallone CA, Barkun AN, Vogt K, Loo V, Trautmann M, Tong JZ, Nguyen TN, Fainsilber T, Hahn HH, et al.: Genetic variability determinants of Helicobacter pylori: influence of clinical background and geographic origin of isolates. I Infect Dis 2000, I8I(5): I674-I68I.

4. Akopyants NS, Eaton KA, Berg DE: Adaptive mutation and cocolonization during Helicobacter pylori infection of gnotobiotic piglets. Infect Immun 1995, 63(I): | |6-I2I.
5. Salaun L, Ayraud S, Saunders NJ: Phase variation mediated niche adaptation during prolonged experimental murine infection with Helicobacter pylori. Microbiology 2005, 15 I (Pt 3):917-923.

6. Guo G, Tong WD, Zeng H, Liu KY, Zou QM: [Comparative proteomics analysis of Helicobacter pylori after adaptive colonization in Mongolian gerbils]. Wei Sheng Wu Xue Bao 2007, 47(3):46|-464.

7. Koga T, Shimada Y, Sato K, Takahashi K, Kikuchi I, Okazaki Y, Miura T, Katsuta M, Iwata M: Contribution of ferrous iron to maintenance of the gastric colonization of Helicobacter pylori in miniature pigs. Microbiological research 2002, I57(4):323-330.

8. Waidner B, Greiner S, Odenbreit S, Kavermann H, Velayudhan J Stahler F, Guhl J, Bisse E, van Vliet AH, Andrews SC, et al.: Essential role of ferritin Pfr in Helicobacter pylori iron metabolism and gastric colonization. Infection and immunity 2002, 70(7):3923-3929.

9. Nakao K, Imoto I, Ikemura N, Shibata T, Takaji S, Taguchi Y, Misaki $M$, Yamauchi K, Yamazaki N: Relation of lactoferrin levels in gastric mucosa with Helicobacter pylori infection and with the degree of gastric inflammation. The American journal of gastroenterology 1997, 92(6): 1005-10I I.

10. Andrews SC, Robinson AK, Rodriguez-Quinones F: Bacterial iron homeostasis. FEMS microbiology reviews 2003, 27(2-3):215-237.

II. Payne SM: Iron acquisition in microbial pathogenesis. Trends in microbiology 1993, I(2):66-69.

12. Wandersman C, Delepelaire P: Bacterial iron sources: from siderophores to hemophores. Annual review of microbiology 2004, 58:611-647.

13. Ridley KA, Rock JD, Li Y, Ketley JM: Heme utilization in Campylobacter jejuni. J Bacteriol 2006, I 88(22):7862-7875.

14. Wyckoff EE, Schmitt M, Wilks A, Payne SM: HutZ is required for efficient heme utilization in Vibrio cholerae. Journal of bacteriology 2004, I 86(13):4I I2-4I5I.

15. Perry RD, Shah J, Bearden SW, Thompson JM, Fetherston JD: Yersinia pestis TonB: role in iron, heme, and hemoprotein utilization. Infection and immunity 2003, 7I(7):4159-4I62.

16. Jarosik GP, Sanders JD, Cope LD, Muller-Eberhard U, Hansen El: A functional ton $B$ gene is required for both utilization of heme and virulence expression by Haemophilus influenzae type b. Infect Immun 1994, 62(6):2470-2477.

17. Wilks A, Burkhard KA: Heme and virulence: how bacterial pathogens regulate, transport and utilize heme. Natural product reports 2007, 24(3):5 I I-522.

18. Frankenberg-Dinkel N: Bacterial heme oxygenases. Antioxid Redox Signal 2004, 6(5):825-834.

19. Worst DJ, Otto BR, de Graaff J: Iron-repressible outer membrane proteins of Helicobacter pylori involved in heme uptake. Infect Immun 1995, 63(10):4161-4165.

20. Worst DJ, Maaskant J, Vandenbroucke-Grauls CM, Kusters JG: Multiple haem-utilization loci in Helicobacter pylori. Microbiology (Reading, England) 1999, I45(Pt 3):68I-688.

21. Wilks A, Schmitt MP: Expression and characterization of a heme oxygenase (Hmu O) from Corynebacterium diphtheriae. Iron acquisition requires oxidative cleavage of the heme macrocycle. The Journal of biological chemistry 1998, 273(2):837-84I.

22. Ishikawa K, Sato M, Yoshida T: Expression of rat heme oxygenase in Escherichia coli as a catalytically active, full-length form that binds to bacterial membranes. European journal of biochemistry/FEBS 1991, 202(1):161-165.

23. Puri S, O'Brian MR: The hmuQ and hmuD genes from Bradyrhizobium japonicum encode heme-degrading enzymes. Journal of bacteriology 2006, 188(18):6476-6482.

24. Merrell DS, Thompson LI, Kim CC, Mitchell H, Tompkins LS, Lee A Falkow S: Growth phase-dependent response of Helicobacter pylori to iron starvation. Infect Immun 2003, 7 I (I I):65 I0-6525.

25. Bereswill S, Greiner S, van Vliet AH, Waidner B, Fassbinder F, Schiltz E, Kusters JG, Kist M: Regulation of ferritin-mediated cytoplasmic iron storage by the ferric uptake regulator homolog (Fur) of Helicobacter pylori. Journal of bacteriology 2000, 182(21):5948-5953.

26. Stahler FN, Odenbreit S, Haas R, Wilrich J, van Vliet AH, Kusters JG Kist M, Bereswill S: The novel Helicobacter pylori CznABC metal efflux pump is required for cadmium, zinc, and nickel resistance, urease modulation, and gastric colonization. Infect Immun 2006, 74(7):3845-3852. 
27. Ratliff M, Zhu W, Deshmukh R, Wilks A, Stojiljkovic I: Homologues of neisserial heme oxygenase in gram-negative bacteria: degradation of heme by the product of the pigA gene of Pseudomonas aeruginosa. J Bacteriol 200I, I 83(2 I):6394-6403.

28. Zhu W, Hunt DJ, Richardson AR, Stojiljkovic I: Use of heme compounds as iron sources by pathogenic neisseriae requires the product of the hemO gene. J Bacteriol 2000, 182(2):439-447.

29. Kunkle CA, Schmitt MP: Comparative analysis of hmuO function and expression in Corynebacterium species. J Bacteriol 2007, I 89(9):3650-3654.

30. Suits MD, Pal GP, Nakatsu K, Matte A, Cygler M, jia Z: Identification of an Escherichia coli O 157:H7 heme oxygenase with tandem functional repeats. Proc Natl Acad Sci USA 2005, I02(47): 16955-16960.

31. Gancz H, Censini S, Merrell DS: Iron and pH homeostasis intersect at the level of Fur regulation in the gastric pathogen Helicobacter pylori. Infect Immun 2006, 74(I):602-6I4.

32. Clayton CLaM, Harry LT: Helicobacter pylori Protocols. Humana Press; 1997.

33. Heuermann D, Haas R: A stable shuttle vector system for efficient genetic complementation of Helicobacter pylori strains by transformation and conjugation. Mol Gen Genet 1998, 257(5):519-528.

34. Michie KA, Monahan LG, Beech PL, Harry EJ: Trapping of a spirallike intermediate of the bacterial cytokinetic protein FtsZ. J Bacteriol 2006, I 88(5): 1680-1690.

35. Lee BC: Isolation of an outer membrane hemin-binding protein of Haemophilus influenzae type b. Infect Immun 1992, 60(3):810-816.

36. Feng Y, Li M, Zhang H, Zheng B, Han H, Wang C, Yan J, Tang J, Gao GF: Functional definition and global regulation of Zur, a zinc uptake regulator in a Streptococcus suis serotype $\mathbf{2}$ strain causing streptococcal toxic shock syndrome. J Bacteriol 2008, I 90(22):7567-7578.

37. Zeng H, Guo G, Mao XH, De Tong W, Zou QM: Proteomic Insights into Helicobacter pylori Coccoid Forms Under Oxidative Stress. Curr Microbiol 2008, 57(4):28I-286.

Publish with Biomed Central and every scientist can read your work free of charge

"BioMed Central will be the most significant development for disseminating the results of biomedical research in our lifetime. "

Sir Paul Nurse, Cancer Research UK

Your research papers will be:

- available free of charge to the entire biomedical community

- peer reviewed and published immediately upon acceptance

- cited in PubMed and archived on PubMed Central

- yours - you keep the copyright 